\title{
Factors affecting hospital length of stay following anterior cervical discectomy and fusion
}

\author{
Authors Paul M Arnold ${ }^{1}$, Lisa R Rice ${ }^{2}$, Karen K Anderson ${ }^{1}$, Joan K McMahon², Lynne M Connelly ${ }^{3}$, \\ Daniel C Norvell ${ }^{4}$ \\ Institution l Department of Neurosurgery, University of Kansas Medical Center, Kansas City, Kansas, USA \\ 2 Department of Nursing, University of Kansas Hospital, Kansas City, Kansas, USA \\ ${ }^{3}$ Department of Nursing, Benedictine College, Atchison, Kansas, USA \\ ${ }^{4}$ Spectrum Research Inc, Tacoma, Washingston, USA
}

\begin{tabular}{|c|c|}
\hline $\begin{array}{l}\text { Final class of evidence } \\
\text { (CoE)-treatment }\end{array}$ & Yes \\
\hline \multicolumn{2}{|l|}{ Study design: } \\
\hline \multicolumn{2}{|l|}{ Prospective cohort } \\
\hline Retrospective cohort & 。 \\
\hline \multicolumn{2}{|l|}{ Case control } \\
\hline \multicolumn{2}{|l|}{ Case series } \\
\hline \multicolumn{2}{|l|}{ Methods } \\
\hline $\begin{array}{l}\text { Patients at similar point in } \\
\text { course of treatment }\end{array}$ & $\circ$ \\
\hline Follow-up $\geq 85 \%$ & 。 \\
\hline $\begin{array}{l}\text { Similarity of treatment } \\
\text { protocols for patient groups }\end{array}$ & $\circ$ \\
\hline $\begin{array}{l}\text { Patients followed-up long } \\
\text { enough for outcomes to occur }\end{array}$ & 。 \\
\hline $\begin{array}{l}\text { Control for extraneous } \\
\text { risk factors }\end{array}$ & $\circ$ \\
\hline Overall class of evidence & II \\
\hline \multicolumn{2}{|c|}{$\begin{array}{l}\text { The definiton of the different } \\
\text { classes of evidence is available on } \\
\text { page } 59 .\end{array}$} \\
\hline
\end{tabular}

\section{ABSTRACT}

Study design: Retrospective cohort study.

Background: Several studies focus on the long-term results of anterior cervical discectomy and fusion (ACDF) surgeries, but little information exists regarding how various patient-related, procedure-related, and payerrelated variables may affect postoperative hospital length of stay (LOS).

Objective: To determine what factors, if any, contribute to increased hospital LOS in patients who have had an ACDF.

Methods: Retrospective cohort study of 108 consecutive patients who underwent elective ACDF at a Midwest academic medical center. Extensive preoperative, intraoperative, and postoperative data were abstracted and analyzed to identify prognostic factors for an increased LOS. Multivariate analysis was performed to analyze the effects of patient and hospital characteristics on hospital LOS.

Results: 103 patients met inclusion and exclusion criteria. The mean LOS for patients undergoing ACDF was 1.98 ( \pm 1.6 ) days. Only $29 \%$ of patients had one level fused. The mean blood loss during surgery was $87.4 \pm 99.6 \mathrm{~mL}$. One subject lost $700 \mathrm{~mL}$ of blood. Complications, though rare, included uncontrolled postoperative pain (13\%), cardiac $(6 \%)$, pulmonary $(4 \%)$, and urinary $(3 \%)$. Covariates included in the final model were age, sex, cardiac complication, urinary complication, and pulmonary complication. Factors that contributed to increased LOS and their associated adjusted mean days were: $\geq 50$ years of age $(2.5 \pm 1.2$ days $)$, female gender $(2.3 \pm 1.2$ days), and three particular types of complications. The complications that had the largest effect on increased LOS from least to most severe were cardiac (3.5 \pm 1.3 days), urinary ( $4.7 \pm 1.3$ days), and pulmonary (5.3 \pm 1.3 days)

The authors have no conflict of interest for this study. No funding was received for research. 
Conclusions: The information presented in this study may be useful for patients, clinicians, and insurance companies, including precertification and case-management services. Our results can be instrumental in designing future prospective studies using more detailed analyses with more patients, more surgeons, and multiple institutions.

\section{STUDY RATIONALE AND CONTEXT}

Anterior cervical discectomy and fusion (ACDF) is indicated for radiculopathy, myelopathy, or neck pain unrelieved by nonsurgical means. Garvey et al [1] found that $78 \%$ of the patients who presented with symptoms attributed them to a motor vehicle injury or work-related injury, leaving $22 \%$ with an unknown etiology of symptoms with no injury involved. Many studies have looked at long-term outcomes of patients who have undergone ACDF, and found a high rate of patient satisfaction [1]. There may be complications, some of which can result in a longer hospital stay; however, we found no studies that included the contribution of perioperative complications to an increased length of stay (LOS). Already identified in other studies are variables that contribute to LOS and recovery time; we analyzed these variables in our study for correlation to LOS, which also plays a role in patient satisfaction and procedure outcome.

\section{OBJECTIVE}

To determine what factors, if any, contribute to increased postoperative hospital stay in patients who have had an ACDF.

\section{MATERIALS AND METHODS}

Study design: Retrospective cohort study using the quota-sampling method for record selection. Records were listed of all patients having had elective ACDF by one attending neurosurgeon, beginning with those who had surgery the month prior to institutional review board application submission. From this list we worked backward with the goal of gathering data from 108 consecutive charts, a cohort number considered adequate to obtain clinically useful results.

\section{Inclusion criteria:}

- Age $\geq 18$ years; nontrauma patient, no other physical injuries

- Admitted to the hospital after surgery

- Unresponsive to at least 6 weeks of conservative treatment and/or presented with progressive symptoms of nerve root or spinal cord compression

- Underwent elective ACDF for: (1) myelopathy, radiculopathy, myeloradiculopathy; (2) stenosis, spinal cord compression, spinal cord change; (3) herniated nucleus pulposus (HNP), degenerative disc disease, spondylosis, osteophytic complexes; and (4) foraminal stenosis

\section{Exclusion criteria:}

- Age < 18 years; trauma patient with other physical injuries

- Emergent admission with nonelective surgery

- Evidence of adjacent segment stiffness secondary to pathology, such as diffuse idiopathic skeletal hyperostosis, ankylosing spondylitis, congenital abnormality, or rheumatoid arthritis

\section{Patient population:}

- A total of 108 consecutive patients underwent elective ACDF surgery by one neurosurgeon; of these, five were not eligible for inclusion (two were involved in trauma; two were emergent admissions with surgery not electively scheduled; and one had osteomyelitis).

\section{Intervention:}

- Anterior approach to perform a discectomy and decompress the affected spinal level. Either autograft or allograft bone was placed in the intervertebral space to stimulate healing and eventual fusion between the end plates. A metal plate was affixed to the adjoining vertebrae to stabilize the construct, maintain neck lordosis, and allow for optimal bone healing and fusion. 


\section{Covariates:}

The following covariates were collected from hospitalbilling records, patient charts, and outpatient clinic records. We were limited to what had been collected.

- Patient demographics (age, sex, weight, height, and type of insurance)

- Medical comorbidities (cardiovascular, previous spine surgery, cancer, central nervous system, central nervous system-psychiatric, gastrointestinal, genitourinary, respiratory, degenerative disc disease/degenerative joint disease, diabetes)

- Tobacco, alcohol, and drug use

- Symptoms: neck pain only, radiating pain, numbness and tingling, weakness, difficulty swallowing/ hoarseness, difficulty with ambulation, and duration of symptoms

- Primary diagnosis in one of five categories:

- myelopathy, radiculopathy, myeloradiculopathy

- stenosis, spinal cord compression, spinal cord change

- HNP, degenerative disc disease, spondylosis, osteophytic complexes

- foraminal stenosis

- previous cervical fusion

- Surgical details (levels of fusion, operative time, estimated blood loss, and preoperative, intraoperative, and postoperative medications)

- Home medications (opioid and nonopioid pain medications, muscle relaxants, steroidal antiinflammatories, non-steroidal antiinflammatories, psychotropic, and other central nervous system medications)

- Payer/insurance characteristics

We noted all postoperative complications reported in the medical record from mild to severe to ensure that any event that could influence hospital LOS was accounted for. The following categories of complications were available: none; new neurological deficit (new onset numbness/weakness in upper extremity (UE); dysphagia; cardiac (which included only hypertension and broadly defined cardiac or noncardiac chest pain); urinary (urinary retention, problems urinating after Foley removal); anxiety; pulmonary (decreased sats, need for $\mathrm{O} 2$ and aggressive pulmonary toilet, exacerbation of asthma, respiratory failure, atelectasis, pneumonia); fever; uncontrolled postoperative pain (severe pain issues, pain uncontrolled by current pain medications, admitted from emergency department for pain, increased need for pain medications).

\section{Outcome:}

- Hospital LOS in days, calculated as date/time of surgery to date/time of discharge.

\section{Analysis:}

- Means \pm standard deviations and frequency counts and rates were reported for continuous and categorical covariates, respectively.

- We performed univariate comparisons of all variables listed in the section covariates to determine our candidate covariates for the final multivariate model. All variables that were associated with the outcome LOS with $P<.05$ were considered in the multivariate model.

- We then built a systematic multivariate linear regression model by adding variables to the model in the order given in the section labeled covariates. Variables that did not have an association of $P<.05$ with LOS did not remain in the model.

- The overall R2, regression coefficients, and associated $P$ values were reported for all covariates included in the final model.

- The adjusted mean LOS and standard deviations for each covariate were computed using the post regression command "adjust" in Stata 9.1. 


\section{RESULTS}

- One hundred and three patients (95.4\%) were deemed eligible and included in the final analysis (Fig 1). There were four missing data elements for insurance information. There were no missing data for other covariates.

- Of 103 patients included in the study, 56 (54\%) were women, and the average age was 49.1 years; average height, 67.3 inches; and average weight, $87.1 \mathrm{~kg}$ (Table 1).

- Sixty-five patients (63\%) had concomitant HNP; 51 $(50 \%)$ had cervical spondylotic myelopathy; and 45 (44\%) had cardiovascular disease; 38 (37\%) of 103 patients who were current cigarette smokers smoked an average of 0.38 packs per day. Other concomitant conditions were stenosis (34\%), psychiatric $(24 \%)$, central nervous system (24\%), gastrointestinal (24\%), previous spine surgery $(17 \%)$, respiratory $(17 \%)$, regular alcohol use $(14 \%)$, diabetes $(12 \%)$, genitourinary $(11 \%)$, cancer $(3 \%)$, and degenerative joint disease (2\%) (Table 1).

- Presenting symptoms included neck pain, 78 patients (76\%); UE pain, 75 (73\%); and unilateral UE numbness, 37 (36\%). Bilateral UE numbness was a presenting symptom in 16 patients $(16 \%)$, as was unilateral UE weakness. Symptom duration was long (12+ months) for 29 patients $(28 \%)$; short (3.0-5.9 months) for 24 patients (23\%); and medium (6.0-11.9 months) for 16 patients (16\%) (Table 2).

\section{Fig 1 Patient sampling and selection.}

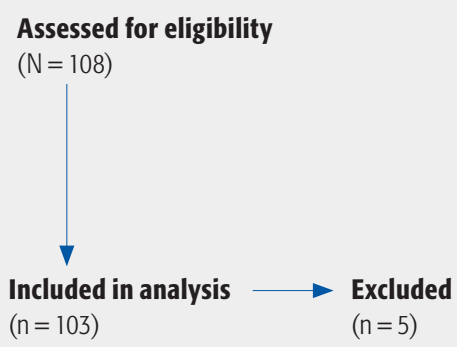

Reasons for exclusion:

- Trauma $(\mathrm{n}=2)$

- Emergent admission, surgery

not electively scheduled $(n=2)$

- Osteomyelitis $(n=1)$
- The mean LOS for patients undergoing ACDF was 1.98 $( \pm 1.6)$ days. Twenty-nine patients $(29 \%)$ had one level fused; 41 , two levels $(41 \%)$; 21 , three levels $(21 \%)$; and 8 , four levels fused $(8 \%)$. The average length of surgery was $3.4( \pm .99)$ hours, and the average blood loss during surgery was $87.4 \pm 99.6 \mathrm{~mL}$. Patients' concomitant medications were tracked, as well as the intraoperative and postoperative medications administered in the hospital (Table 3).

- Individual perioperative complications were rare, the most common of which were uncontrolled postoperative pain (severe pain issues, pain uncontrolled by current pain medications, admitted from the emergency department for pain, increased need for pain medications: $13 \%$ ), as well as cardiac complications (which included only hypertension and broadly defined cardiac or noncardiac chest pain: $6 \%$ ), urinary complications (urinary retention, problems urinating after Foley removal: $3 \%$ ), and pulmonary complications (decreased sats, need for $\mathrm{O} 2$ and aggressive pulmonary toilet, exacerbation of asthma, respiratory failure, atelectasis, pneumonia: 4\%) (Table 4). Most complaints of chest pain were due to neck muscles pulling on the chest wall; no actual cardiac events occurred postoperatively as verified by cardiac work-up. There were no procedure-related or wound complications, and this study did not track delayed complications in the outpatient setting.

- The following covariates had an association of $P<.05$ and were included in the final multivariate regression model for hospital LOS (in days) as outcome: (Table 5)

- Age: dichotomized at 50 years (coefficient $=.851$; $P=.001)$

- Sex: $($ coefficient $=-.620 ; P=.011)$

- Cardiac complication (coefficient $=1.58 ; P=.002$ )

- Urinary complication (coefficient $=2.77 ; P<.001$ )

- Pulmonary complication (coefficient $=3.45$; $P<.001)$

- The overall multivariate model $\mathrm{R}^{2}$ was .48. Factors that contributed to increased LOS and their associated adjusted mean days were age $\geq 50$ years $(2.5 \pm 1.2$ days), female gender ( $2.3 \pm 1.2$ days), and three particular types of complications. The three types of complications that had the largest effect on increased LOS, from least to most severe, were: cardiac ( $3.5 \pm 1.3$ days); urinary $(4.7 \pm 1.3$ days); and pulmonary $(5.3 \pm 1.3$ days $)$ (Table 5).

- No other factors listed in the covariates section in Tables 1-4 were found to be significantly associated with hospital LOS. The presence of cervical spondylotic myelopathy was approaching significance $(P=.068)$. 
Table 1 Baseline demographics and general health characteristics of patients undergoing cervical fusion*.

\begin{tabular}{ll}
\hline Characteristic & $\begin{array}{l}\text { Patients (N= 103), } \\
\text { No. (\%) or mean } \pm \text { SD }\end{array}$ \\
\hline Female & $56(54)$ \\
\hline Age, y & $49.1 \pm 9.9$ \\
\hline Height, in & $67.3 \pm 4.1$ \\
\hline Weight, kg & $87.1 \pm 19.1$ \\
\hline Smoke, packs per day & $.38 \pm .60$ \\
\hline Regular alcohol use, yes & $14(14)$ \\
\hline Previous spine surgery & $17(17)$ \\
\hline Psychiatric issues & $25(24)$ \\
\hline Respiratory & $17(17)$ \\
\hline Degenerative joint disease & $2(2)$ \\
\hline Cervical spondylotic myelopathy $\dagger$ & $51(50)$ \\
\hline Herniated nucleus pulposus $\ddagger$ & $65(63)$ \\
\hline Stenosis & $35(34)$ \\
\hline Central nervous system & $25(24)$ \\
\hline Diabetes & $12(12)$ \\
\hline Gastrointestinal & $25(24)$ \\
\hline Cardiovascular disease & $45(44)$ \\
\hline Genitourinary & $11(11)$ \\
\hline Cancer & $3(3)$ \\
\hline
\end{tabular}

* Missing 11 height and 2 weight data points.

† Including myelopathy, radiculopathy, and myeloradiculopathy.

‡ Herniated disc, degenerative disc disease, spondylosis, osteophytes.

Table 2 Baseline pain and symptoms of patients undergoing cervical fusion*.

\begin{tabular}{ll}
\hline Characteristic & Patients (N=103), No. (\%) \\
\hline Neck pain & $78(76)$ \\
\hline UE pain & $75(73)$ \\
\hline Unilateral UE numbness & $37(36)$ \\
\hline Bilateral UE numbness & $16(16)$ \\
\hline Unilateral UE weakness & $16(16)$ \\
\hline Bilateral UE weakness & $3(3)$ \\
\hline Unilateral LE numbness & $5(5)$ \\
\hline Bilateral LE numbness & $2(2)$ \\
\hline Unilateral LE weakness & $3(3)$ \\
\hline Bilateral LE weakness & $3(3)$ \\
\hline Ambulation difficulty & $5(5)$ \\
\hline Symptom duration (short) & $24(23)$ \\
\hline Symptom duration (medium) & $16(16)$ \\
\hline Symptom duration (long) & $29(28)$ \\
\hline
\end{tabular}

* UE indicates upper extremity; LE, lower extremity.
Table 3 Surgery-specific characteristics of patients undergoing cervical fusion*.

\begin{tabular}{|c|c|}
\hline Factor & $\begin{array}{l}\text { Patients }(\mathrm{N}=103) \text {, } \\
\text { No. }(\%) \text { or mean } \pm \mathrm{SD}\end{array}$ \\
\hline Length of hospital stay, d & $1.98 \pm 1.6$ \\
\hline Length of surgery, $h$ & $3.4 \pm .99$ \\
\hline \multicolumn{2}{|l|}{ Levels fused } \\
\hline 1 & $29(29)$ \\
\hline 2 & $41(41)$ \\
\hline 3 & $21(21)$ \\
\hline 4 & $8(8)$ \\
\hline Blood loss, mL & $87.4 \pm 99.6 \dagger$ \\
\hline \multicolumn{2}{|l|}{ Intraoperative medications } \\
\hline Anti-infectives & $97(94)$ \\
\hline Cardiovascular & $46(45)$ \\
\hline NSAIDS & $4(4)$ \\
\hline Steroids & $54(52)$ \\
\hline \multicolumn{2}{|l|}{ Postoperative medications } \\
\hline Pain IV & $20(19)$ \\
\hline Pain narcotic & $101(98)$ \\
\hline Pain non-narcotic & $77(75)$ \\
\hline Muscle relaxant & $27(26)$ \\
\hline Sedatives & $18(18)$ \\
\hline Pain consult & $32(31)$ \\
\hline \multicolumn{2}{|l|}{ Home medications } \\
\hline Opioids & $51(50)$ \\
\hline Pain nonopioid & $20(19)$ \\
\hline Muscle relaxants & $27(26)$ \\
\hline Steroids & $5(5)$ \\
\hline NSAIDs & $32(31)$ \\
\hline Psychotropics & $45(44)$ \\
\hline Central nervous system & $32(31)$ \\
\hline
\end{tabular}

* NSAIDS indicates nonsteroidal antiinflammatory drugs; IV, intravenous.

† One patient had $700 \mathrm{~mL}$ blood loss and was treated with iron tid.

Table 4 Postoperative complications after cervical spine fusion.

\begin{tabular}{ll}
\hline Complications & Patients $(\mathbf{N}=\mathbf{1 0 3})$, No. (\%) \\
\hline Any complication & $77(75)$ \\
\hline Neurological & $1(1)$ \\
\hline Dysphagia & $2(2)$ \\
\hline Cardiac & $6(6)$ \\
\hline Urinary & $3(3)$ \\
\hline Anxiety & $1(1)$ \\
\hline Pulmonary & $4(4)$ \\
\hline Fever & $2(2)$ \\
\hline Pain & $13(13)$ \\
\hline
\end{tabular}


Table 5 Multivariate linear regression analysis of length of stay (in days) as outcome with all covariates that have an association of $P<.05$.

\begin{tabular}{|c|c|c|c|}
\hline Covariate & $\begin{array}{l}\text { Mean Days, * } \\
\pm \text { SD }\end{array}$ & $P$ value* & Model $\mathbf{R}^{2}$ \\
\hline \multicolumn{4}{|l|}{ Age, y } \\
\hline$\leq 50$ & $1.6 \pm 1.2$ & \multirow[t]{2}{*}{.001} & \multirow[t]{2}{*}{.48} \\
\hline$>50$ & $2.5 \pm 1.2$ & & \\
\hline \multicolumn{4}{|l|}{ Sex } \\
\hline Male & $1.6 \pm 1.2$ & \multirow[t]{2}{*}{.011} & \\
\hline Female & $2.3 \pm 1.2$ & & \\
\hline \multicolumn{4}{|c|}{ Cardiac complication } \\
\hline No & $1.8 \pm 1.2$ & \multirow[t]{2}{*}{.002} & \\
\hline Yes & $3.2 \pm 1.3$ & & \\
\hline \multicolumn{4}{|c|}{ Urinary complication } \\
\hline No & $1.9 \pm 1.2$ & \multirow[t]{2}{*}{$<.001$} & \\
\hline Yes & $4.7 \pm 1.3$ & & \\
\hline \multicolumn{4}{|c|}{ Pulmonary complication } \\
\hline No & $1.8 \pm 1.2$ & \multirow[t]{2}{*}{$<.001$} & \\
\hline Yes & $5.3 \pm 1.3$ & & \\
\hline
\end{tabular}

${ }^{*}$ Adjusted for other variables in the model.

\section{DISCUSSION}

- Individuals at our facility who had identical surgeries by the same surgeon were found to have very different LOS, with no clear-cut explanation for the difference. Most patients were discharged only one night after surgery, but others were discharged as long as five nights after surgery. We sought to identify the factors that contribute to increased LOS in postoperative patients having had ACDF, and to develop a plan to counteract and anticipate these factors to reduce LOS and to improve overall outcomes.

- The mean LOS for patients undergoing ACDF was 1.98 $( \pm 1.6)$ days. Complications, though rare, included uncontrolled postoperative pain (13\%), cardiac $(6 \%)$, pulmonary $(4 \%)$, and urinary (3\%). Because our study was not to determine overall safety of a procedure or device but rather to determine what types of events influence hospital LOS, we chose to document any event that could lead to an increased LOS, with the understanding that the event may not be considered severe. Therefore, the percentage of complications may be overestimated compared with those in other studies because our operational definitions had to be broad.

- Factors that contributed to increased LOS and their associated mean days were age $\geq 50$ years, female gender, and three particular types of complications. The complications that had the largest effect on increased LOS from least to most severe were cardiac, urinary, and pulmonary.
- Castro et al [2] noted that dysphagia indicated additional time in the hospital postoperatively, but there was no indication to how much more time. This particular study was the only reference to LOS that was found. Compared with the results of Castro et al, the rate of dysphagia in our study was lower, which we attribute to meticulous surgical technique as well as deflation of the endotracheal cuff during surgery. Studies that have reviewed the long-term outcomes of ACDF surgeries list complications, but none discussed how those complications contribute to an increased LOS. Garvey et al [1] concluded that it was not possible to determine why individuals with the same anatomical findings or pathology continued to have symptoms after surgery, while others did not.

- Factors unrelated to the medical condition can influence hospital LOS, such as type and location of hospital, type of payer, and patient characteristics [3-6].

- Factors related to the patient and condition that can influence outcome after cervical spine surgery include: comorbidity [7-10]; age, severity of the neurological deficit [10]; preoperative myelopathy [11-13]; pulmonary and cardiovascular disease, hypertension, and diabetes mellitus [12,14]; smoking, alcohol use, body mass index [11]; education level, length of surgery, number of fusion levels, dysphagia, incisional pain, urinary tract infection, septicemia, bleeding, and hypotension $[2,15,16]$.

- Clinical myelopathy, age, and comorbidity are associated with a longer hospital LOS and critical care intervention after cervical spine surgery [7, 8, 12,17]. In our study, the presence of cervical spondylotic myelopathy was approaching significance $(P=.068)$, but was found not to be significantly associated with hospital LOS, therefore not included in the final model and estimates.

- Higher rates of complication are expected in patients having multilevel rather than single-level fusion because of more extensive dissection, longer operating time, and greater blood loss [17].

- In patients who have undergone surgical decompression for cervical degenerative disc disease, the factors associated with prolonged hospitalization and the need for postoperative critical care are preexisting myelopathy, multilevel decompression, cardiovascular disease, hypertension, pulmonary disease, and diabetes mellitus [12].

\section{Limitations of this study}

- It is difficult to establish cause-and-effect using retrospective analysis. Such findings are subject to confounding bias because of known or unknown factors that influence outcomes that are not controlled. We collected as many variables as we could that we believed might have an impact on outcome. 
- Retrospective studies are known for missing data, which can affect study validity. Only insurance data was missing in our analysis $(n=4)$ and these variables were not statistically significant.

- Since these data were not collected prospectively, there is also the possibility of misclassification of admitting diagnosis and other covariates included in the analysis. Such misclassification would likely not be systematic and therefore considered nondifferential. Nondifferential misclassification generally biases associations toward the null.

- Because we were predicting outcome after a singletreatment technique and not comparing treatment techniques, the effects of selection bias may influence generalizability (as opposed to biasing a treatment). That is, our patients may not be representative of the typical patients at other centers. That said, we enrolled a consecutive series of patients meeting the study criteria and we believe our study population represented the normal patient population that presents for ACDF.

- Even the process of interpreting and abstracting data from hospital charts and outpatient records creates potential variability in the collected data, even if abstracted by only one researcher [18]. The quality of the information recorded may vary; however, there is no reason to believe this would be systematic.

\section{SUMMARY AND CONCLUSION}

- We found that for patients who have undergone ACDF, the prognostic factors that contribute to increased postoperative hospital LOS are older age, female gender, and three types of complications-cardiac, urinary, and pulmonary.

- The information presented in this study may be useful for patients, clinicians, and insurance companies, including precertification and case-management services. Our results can be instrumental in designing future prospective studies using more detailed analyses with more patients, more surgeons, and multiple institutions. A prediction model using registry data could also be performed to validate or refine these observations.

\section{REFERENCES}

1. Garvey TA, Transfeldt EE, Malcolm JR, et al (2002) Outcome of anterior cervical discectomy and fusion as perceived by patients treated for dominant axial-mechanical cervical spine pain. Spine; 27(17):1887-1895.

2. Castro FP Jr, Holt RT, Majd M, et al (2000) A cost analysis of two anterior cervical fusion procedures. J Spinal Disord; 13(6):511-514.

3. Cahill KS, Chi JH, Day A, et al (2009) Prevalence, complications, and hospital charges associated with use of bone-morphogenetic proteins in spinal fusion procedures. JAMA; 302(1):58-66.

4. Brasel KJ, Lim HJ, Nirula R, et al (2007) Length of stay: an appropriate quality measure? Arch Surg; 142(5):461-465.

5. Rosenthal GE, Harper DL, Quinn LM, et al (1997) Severity-adjusted mortality and length of stay in teaching and nonteaching hospitals: results of a regional study. JAMA; 278(6):485490.

6. Schmidt WP, Taeger D, Buecker-Nott HJ, et al (2003) The impact of the day of the week and month of admission on the length of hospital stay in stroke patients. Cerebrovasc Dis; 16(3):247-252.

7. Librero J, Peiró S, Ordiñana R (1999) Chronic comorbidity and outcomes of hospital care: length of stay, mortality, and readmission at 30 and 365 days. J Clin Epidemiol; 52(3):171-179.

8. Rochon PA, Katz JN, Morrow LA, et al (1996) Comorbid illness is associated with survival and length of hospital stay in patients with chronic disability: a prospective comparison of three comorbidity indices. Med Care; 34(11):1093-1101.

9. Boakye M, Patil CG, Santarelli J, et al (2008) Cervical spondylotic myelopathy: complications and outcomes after spinal fusion. $\mathrm{Neu}$ rosurgery; 62(2):455-461.

10. Malik SA, Murphy M, Connolly P, et al (2008) Evaluation of morbidity, mortality and outcome following cervical spine injuries in elderly patients. Eur Spine J; 17(4):585-591.

11. Shamji MF, Cook C, Tackett S, et al (2008) Impact of preoperative neurological status on perioperative morbidity associated with anterior and posterior cervical fusion. $J$ Neurosurg Spine; 9(1):10-16.

12. Harris OA, Runnels JB, Matz PG (2001) Clinical factors associated with unexpected critical care management and prolonged hospitalization after elective cervical spine surgery. Crit Care Med; 29(10):1898-1902.

13. Wang MC, Chan L, Maiman DJ, et al (2007) Complications and mortality associated with cervical spine surgery for degenerative disease in the United States. Spine; 32(3):342-347. 
14. Kawaguchi $Y$, Kanamori $M$, Ishihara $H$, et al (2003) Pathomechanism of myelopathy and surgical results of laminoplasty in elderly patients with cervical spondylosis. Spine; 28(19):2209-2214.

15. Cauthen JC, Kinard RE, Vogler JB, et al (1998) Outcome analysis of noninstrumented anterior cervical discectomy and interbody fusion in 348 patients. Spine; 23(2):188-192.

16. Klein GR, Vaccaro AR, Albert TJ (2000) Health outcome assessment before and after anterior cervical discectomy and fusion for radiculopathy: a prospective analysis. Spine; 25(7):801803.

17. Shamji MF, Cook C, Pietrobon R, et al (2009) Impact of surgical approach on complications and resource utilization of cervical spine fusion: a nationwide perspective to the surgical treatment of diffuse cervical spondylosis. Spine J; 9(1):31-38.

18. Connelly LM (2008) Retrospective chart reviews. Medsurg Nurs; 17(5):322-323.

\section{EDITORIAL PERSPECTIVE}

In this new era of quality-of-care concerns and focus on outcomes, the reviewers congratulate Arnold and colleagues for taking on an important subject of complications related to a 'routine' procedure and providing a clear and objective reflection on variables of outcomes. By not being selective but rather inclusive of all patients this retrospective study, this study of grade 3 or less ranking on the evidence-base pyramid actually serves a more valuable purpose than a grade 1 prospectively randomized clinical trial. All patients treated with a similar procedure for a similar condition were documented in a registrystyle undertaking and to the best ability of the authors relevant data points were recorded.

The reviewers and the authors both agreed that certain data points, such as cardiac events, were probably over reported due to lack of clear operational definitions; whereas others, such as swallowing difficulties, were likely under reported due to lack of study focus and follow-up on this entity.

The reviewers applauded the honesty of the authors and noted the impressive variance of something simple, such as length of stay after routine anterior neck surgery, as well as the difficulty in identifying simple answers to try to prevent complications or potentially decrease length of stay through planned interventions.

From an editorial point of view, this article illustrates an important point: as we enter an era of metrics ubiquitously applied to all aspects of healthcare under the guise of 'rewarding quality' we have to realize just how complex our lives are - the variables that influence the length of stay following a simple anterior neck surgery are as perplexing as human life itself. That said, studies such as these are needed to create more meaningful care maps, set realistic expectations with patients and help us represent the complexity of our work better with insurers and regulatory agencies. 